\title{
Identity Projection Strategies for Non-Focal Actors in Digital Ecosystems
}

\author{
Shiyuan Liu \\ Warwick Business School \\ $\underline{\text { shiyuan.liu.17@,mai.wbs.ac.uk }}$
}

\author{
Ola Henfridsson \\ University of Miami \\ ohenfridsson@miami.edu
}

\author{
Joe Nandhakumar \\ Warwick Business School \\ joe.nandhakumar@wbs.ac.uk
}

\begin{abstract}
In digital ecosystems, non-focal actors cannot survive without working on the identity of their apps. The identity expresses what the app is about to customers. However, it also projects an image of the role of the non-focal actor in the larger ecosystem. Such identity projection is relevant for managing the relationship with focal actors of the digital ecosystem. We outline and test three strategies for identity projection (identity conformity, identity differentiation, and identity refinement). Using panel data of social networking applications in the iOS appStore in China between 2014 and 2019, we investigate the influence of non-focal actors' identity projection on their survival in digital ecosystems. Our result shows significantly increased app survival for those who actively pursue the identity projection strategies in three directions. Thus, we shed light on the role of platform identity in navigating platform competition in the digital age when participation across ecosystems becomes compulsory for every digital business.
\end{abstract}

\section{Introduction}

Non-focal actors that grow upon a platform ecosystem confront a thorny challenge. A strong commitment to a single native ecosystem will expose the actor to local failure risk (e.g. user outflow, ecosystem collapse), sometimes leading to a domino effect that pulls each other down [1]. Furthermore, as the focal positions of a few well-established platform incumbents have been entrenched in the market with advanced digital infrastructures and unparalleled network effects, the room for growth in each segment becomes extremely limited, even for those with superior technology and performance $[2,3]$. In this regard, young non-focal actors will be less likely to survive due to the inability to rapidly reach a minimum level of scale [4].

For all these reasons, participation across platform ecosystems has increasingly became a necessity for the non-focal actor to survive in digital markets $[5,6]$. Specifically, non-focal actors become tolerant of external shocks or catastrophic events in their native ecosystems, increasing their chance of survival [1]. At the same time, cross-ecosystem participation makes it possible to leverage as many potential user bases as possible [7], access external knowledge and capability [6], and expand innovation scope with low cost $[6,8]$. As such, a non-focal actor is able to continually explore new growth opportunities beyond the native ecosystem, which is argued to be vital for its survival and thriving [3].

Despite the apparent benefits of cross-ecosystem participation for the survival of non-focal actors, it might come with significant friction. In particular, to survive and take root in an ecosystem, a non-focal actor needs legitimacy, that is, endorsement by powerful external stakeholders [9] such as focal platforms and end-users $[10,11]$. For instance, in order to fully take advantage of the infrastructure resources and network resources in an ecosystem, non-focal actors will incur considerable technical or commercial cost (e.g. adopt focal platforms' language) to conform to its specifications [7, 12]. Moreover, many of non-focal actors fail to match between their products and user demand in the ecosystem within a limited time [13], leading to an incompetent perception from consumers that they are unable to provide viable product enhancement and support [3].

To address these legitimacy issues, non-focal actors might carve out and convey an attractive, desired identity —also known as "identity projection" [14] in terms of who they are and what they do in an ecosystem. Identity projection is a way through which the non-focal actor communicates its desirability, appropriateness and competence relative to other non-focal actors [15]. In this regard, identity is particularly prominent for survival of non-focal actors through facilitating legitimacy acquisition under the context of high uncertainty and ambiguity [16]. In particular, by projecting identity consistent with a particular strategic group operating in the industry, a non-focal actor can quickly share similar competition gene of members (e.g. available resources, cognitive factors, market conditions) which facilitates their viability with lower adjustment cost and shorter adjustment time [17, 18, 2]. 
Meanwhile, by projecting identity distinct from within group members, a non-focal actor is more likely and easier to stand out from the competition for stakeholders' support $[19,9,20]$.

To date, however, there has been sparse inquiry into the role of identity projection in navigating non-focal platform survival in digital ecosystems. To fill the limited insights into the effects of identity projection on platform survival in digital ecosystems, we draw on platform and identity literature (e.g. $[21,14,6]$ ) to explore strategies to cross-ecosystem participation for non-focal actors. Hence, we address the following question: What are the effects of identity projections on the survival of non-focal actors in digital ecosystems?

To answer this question, we use data from the iOS appstore in China to empirically test the influence of non-focal platforms' identity projection strategies on their probability of survival. We focus on social applications between 2014 and 2019, during which the annual growth rate of mobile app market in China dropped below $3 \%$ and numerous existing and emerging apps across different sub-markets decided to participate in the social networking ecosystem for exploring new growth opportunities. As one of the key challenges, those non-focal actors confront a specific identity issue - should the identity of the platform be projected more as a social app or as non-social app in order to be competitive in this ecosystem? Our result shows that app developers need to actively enhance and maintain their identity's both conformity to focal actors' expectation and distinctiveness within a defined membership in order to be competitive and survive in the social networking ecosystem.

Our research contributes to the platform literature, especially platform identity, by conceptualizing three identity projection strategies that non-focal platforms can adopt to navigate their escalating competition in digital ecosystems. Combining the three strategies, this paper also lifts a tip of the dynamic nature of platform identity, which lays the foundation for the future investigation of identity in a digital world.

\section{Focal and Non-Focal Actors in Platform Ecosystems}

A platform ecosystem can be seen as "a collective of firms that is inter-linked by a common interest in the prosperity of a digital technology for materializing their own product or service innovation" ([6], pp. 184-185). This definition implies at least three things. First, in platform-based ecosystems, actors have to draw upon each other's resources and capabilities to enact their agency $[22,23]$ due to the self-referential attribute [24] of digital technologies, where digital innovation requires to build on digital technologies and so on (cf.
[25]). Second, actors in the ecosystem target at a common value proposition-that is, the promised benefit that is to receive in the effort of prospering a digital technology. This collective value proposition not only defines the boundary of the relevant ecosystem, but also requires to reach a mutual agreement among actors in terms of their positions and activity flow among them in the ecosystem [26]. As such, platform ecosystem represents a defined alignment structure that different actors support and defend. Finally, while actors' membership is defined in the ecosystem, it does not mean uncontested, unvarying or complete. Instead, different actors may have their own end goals and end states in mind. For instance, a failure to acquire anticipated capabilities and knowledge in an ecosystem may trigger the actors to leave it [6]. Consequently, they may not commit to a specific ecosystem but continuously (re)assess their engagement across different ecosystems.

Despite being inter-linked, ecosystem actors are not of equal weight $[5,27]$. In this regard, we adopt the distinction between focal actors and non-focal actors (cf. [6]). First, focal actors are characterized of the innovation hub in a digital ecosystem, which design at least one foundation technology or service that is essential for a broader technological system and market. In this regard, it is the foundation on which other actors build their innovation [28]. Further, focal actors have the capability of managing the overall positive network effects (e.g. nourish participant diversity and interaction) in the ecosystem [29]. As such, focal actors are those platforms that have considerable influence over the livelihood of remaining actors and define the forward evolution of the ecosystem [19]. It should be noted that the innovation hub in a platform ecosystem could be multipolar. For example, Microsoft and Sony are both focal actors in the game console ecosystem. Second, non-focal actors are the majority of ecosystem participants who are at the peripheral of a digital ecosystem. They do not hold such core elements (e.g. technology, product or service) or network central position that will influence a large proportion of parts of the ecosystem. Therefore, the ecosystem's survival does not depend on any single non focal actor's participation, but non-focal actors' innovation and development have to draw on the support provided by the focal platforms. This comprises a one-way unique dependency in the sense that a particular non-focal actor cannot function without focal actors, but not vice versa.

Acknowledging the power asymmetry among ecosystem participants, it is therefore necessary for a non-focal actor to seek support from focal actors in order to be competitive in digital ecosystems. Specifically, as non-focal actors are restricted to the domain expertise, sector knowledge and locally relevant 
solutions $[8,6]$, their capability of expanding product's functionality and meaning has to build upon focal actors' infrastructure resources, which otherwise would be inaccessible within their own innovation space [30, 24]. Similarly, as focal actors create and capture the majority value derived from network effects in the ecosystem [29], non-focal actors have to compete for their attention in order to take advantage of their distribution resources (e.g. user base) and monetization means which otherwise would be inaccessible within their own innovation space $[31,6]$. To best leverage the external capabilities and resources offered by various focal actors, non-focal actors tend to actively search and participate in multiple ecosystems in a cherry-picking fashion $[32,30]$.

\section{Platform Identity Projection}

To gain the endorsement of focal actors (both platform owners and end-users), non-focal actors seek to develop its legitimacy in the ecosystem. According to Suchman [33], legitimacy is the perception that an actor is "desirable, proper, or appropriate within some socially constructed system" (pp. 574). In our context, this system represents the platform ecosystems in which the non-focal actor operates and from which it needs resources for survival and growth. As such, the motivating factor for focal actors in that ecosystem to give such resources is their belief or feeling that the nonfocal actor is indeed competent, worthy, needed, efficient or effective (e.g. [10, 11]). At the key to influence such social judgment in the eye of the beholders, the non-focal actor must (re)project its identity strategically which facilitates to gain and maintain a favorable position in the ecosystem [14].

Defining essential features (i.e. key values, products, services, or practices) central to the organization's character [20], identity aids digital enterprises to enact and express who they are and what they do in an ecosystem. For platform businesses, it delineates both platform architecture which depicts the technological capability of a platform, and the way platform technological components function and connect platform participants, and platform scope which depicts the market positioning of the platform along the map of the consumers' preferences and relative to other actors in an ecosystem [21].

In particular, by projecting [14] the identity within a membership of broad groups of similar organizations, a non-focal platform is able to align itself with the takenfor-granted, already-legitimated structure and practices which mirror actors' expectations about its "appropriate" and "inappropriate" positions and activities in the ecosystem $[17,11]$. As such, legitimacy is achieved through identity conformity which demonstrates its consistency or conformity to the institutionalized preferences [34].

At the same time, by projecting the identity different enough from the exemplars (or prototypes) in the ecosystem, a non-focal platform can convey its novelty or distinction with respect to the competence of making unique contribution towards the innovation potential or network effects in the ecosystem. As such, legitimacy is achieved through identity differentiation which demonstrates its distinctiveness which are preferred by external stakeholders [9] and make it more likely to win the competition for the focal platforms' attention.

In the end, a platform identity is more likely to be judged as plausible by focal actors in the ecosystem when it is legitimately distinctive-that is, it "incorporates institutionalized beliefs in ways that introduce novelty but still evidence some conformity" ([9], pp. 480). This corresponds to Brewer's [35] "optimal distinctiveness", wherein actors' identity is different enough from others in a category but still not so different that it is seen as a member in other categories. In other words, a non-focal platform has to project an identity that is perceived as a "good risk" by focal actors in order to realize its survival prospects and growth trajectories. On the one hand, identity differentiation helps generate interest and commitment from focal actors by connecting broader ecosystem context in such a way that the proposed endeavor seems distinctive and original. On the other hand, this distinctive identity has to maintain the soundness through identity conformity which reduces the perceived uncertainty associated with its exploitation of the ecosystem resources in such a way that the threat of undermining the position of focal actors is under control.

However, developing such legitimately distinctive identity does not necessarily signify that it can be maintained by the non-focal platform in the long term. This uncertainty and pressure of sustaining projected identity are particularly salient in digital markets, where legitimacy is highly contested by platform actors who are aggressively competing to institute their offerings, market, and position [36, 37], and establishing their credibility and authority in ecosystems $[38,31]$ due to the winner-take-all logic [18]. As a result, non-focal platforms have to prevent from losing their established legitimacy in the face of emerging ecosystem participants through identity refinement which refers to the reinforcement and clarification of projected identity without major revision or reformulation [39]. All in all, non-focal platform actors must deliberately project its identity in terms of identity conformity, identity differentiation and identity refinement in order for acquiring and maintaining legitimacy in digital ecosystems. 


\section{Hypothesis Development}

As discussed above, extant research suggests a strong association between identity projection and nonfocal actor development in platform ecosystem context. Specifically, we propose following five hypotheses to test the effect of different identity projection strategies on non-focal platform survival in digital ecosystems.

\subsection{Identity conformity}

As one of the key strategies to gain legitimacy, nonfocal actors demonstrate its conformity to the taken-forgranted, already-legitimated structure in the social system [11]. In platform ecosystems, it enacts in the defined common interest and aligned inter-linkage (e.g. focal and non-focal position) among ecosystem actors. In this regard, there are at least two specific strategies that a non-focal actor can adopt to achieve such conformity.

First, connecting with boundary resources (e.g. API) provided at focal platforms, a non-focal actor can not only access to superior innovation capability and network resources $[8,31]$, but also communicate its non-focal position in the ecosystem with increasing dependency on focal platforms. As such, boundary resource connection activities help the actor to gain the legitimacy from focal platform owners with regard to its competence in contributing to the ecosystem and appropriateness in deferring to the leader positions of focal platforms. Consequently, we derive the following hypothesis:

H1(a): Boundary resource connection is positively associated with the likelihood of the survival of nonfocal app in digital ecosystems.

Second, at the same time, a newly joined actor of a digital ecosystem can share similar strategic group gene through developing platform architecture similar to other ecosystem members [2]. While such an architecture assimilating process helps the actor to commit to a membership of broad groups of like organizations in the ecosystem, it increasingly blurs the actor's characteristics comparing with incumbent nonfocal members, leading to a less competitive position when rivaling for the attention of focal platform owners and end-users. This is especially salient in mature markets within which most consumer demands are served by incumbent non-focal actors who have established their position in the particular ecosystem. As such, the contribution of architecture assimilating to actor development is expected to be limited in digital ecosystems. We therefore formulate the following hypothesis:
H1(b): Architecture assimilation does not significantly increase the likelihood of the survival of non-focal platforms in digital ecosystems.

\subsection{Identity differentiation}

At the same time, extant research recognizes the potential to build legitimacy through demonstrating the actor's novelty, distinctiveness or nonconformity [9]. However, such nonconformity has to be perceived as "good risk" by focal platforms in the sense that it is not threating their focal position in the ecosystem. To this end, the non-focal actor has to differentiate itself from other similar actors within the ecosystem and sticks to its non-focal positioning at the same time. There are at least two specific strategic activities to achieve this purpose.

First, through successively dissimilating the platform architecture from others affiliating in same membership, the non-focal actor is able to build an asymmetric identity domain when competing with other incumbent non-focal members. It is not only attractive for the end-users at focal platforms, but also helpful for gaining legitimacy from focal platforms given their dissimilar architecture through leveraging different and unique platform capabilities. In particular, since focal platforms will only react to others' action in a particularly salient "competitive arena that best demonstrates and reinforces their identity in the marketplace" ([40], pp. 48), their endorsement is more likely to sustain as long as the actor keeps asymmetric identity domain. Consequently, we propose:

H2(a): Architecture dissimilation is positively associated with the likelihood of the survival of nonfocal platforms in digital ecosystems.

Second, actors can materialize the identity differentiation by directly adjusting its membership affiliation and corresponding user group in the ecosystem. While such membership shift helps to revitalize existing platform offerings in new market scope, it may highly confuse and decrease the identity recognition from existing ecosystem members (e.g. onboard app users, app cooperators) in terms of who it is and what it does, leading to negative feedback from relevant stakeholders [14] and less effectiveness of growth strategies at the non-focal platform [41]. For those reasons, we formulate:

H2(b): Membership shift does not significantly increase the likelihood of the survival of non-focal platforms in digital ecosystems.

\subsection{Identity refinement}

Lastly, the actor is able to maintain its legitimacy in the ecosystem through refining its projected identity. 
Specifically, the distinctive attributes and membership conformity established before at platform can be further sharpened to reinforce its ecosystem position [39]. In mobile app markets, this can be achieved by on-going optimization of existing platform architecture and API connection which defines its current membership and competence [21], Thus, we formulate:

H3: Architecture maintenance is positively associated with the likelihood of the survival of nonfocal platforms in digital ecosystems.

\section{Research Method}

\subsection{Empirical setting}

Our empirical focus is on the mobile app market in China. Starting from the early twenty-first century, China' mobile app market experienced a high-growth period with the popularization of $3 \mathrm{G}$ and $4 \mathrm{G}$ infrastructure. After the total mobile internet users grew to more than 500 millions in 2014, China Internet Network Information Centre (CNNIC) reported a slowdown of the yearly growth rate for the first time, following by an on-going decline to less than $10 \%$ until 2019. This saturation trend of market further manifested in the dominant position of platform giants including Tencent, Alibaba, Baidu and Sina. Controlling about $80 \%$ of the user base and app use time in the market, the four giants developed all-embracing platform ecosystems with unmatched competition barriers.

As the market became stable and concentrated on a few platform giants, more emerging and existing actors are forced to be more aggressive by exploiting new growth opportunities across multiple dominant ecosystems. In particular, by combining social computing features in core value interaction, these nonfocal actors could further leverage the value creation and capture capabilities at apps in terms of user engagement, retention and monetization $[2,42]$ which lead to a sustainable competitive position in the matured market. Typical examples include Pinduoduo which combines e-commerce with social network [43], Douyin which combines short video with social network [44], and NetEase Cloud music which combines music content with social community [42]. Given the increasing diversity and universality of apps joining in this social networking ecosystem from 2014, we choose it as the basis for following data collection and analysis.

\subsection{Data collection}

In the first step, we screened the apps at apple's iOS store in China between Jan 2014 and July 2019 from a third-party app statistic platform Analysys. We omitted long-tail apps that were not counted by Analysys due to their short life cycle less than one month and unstable monthly user base lower than ten thousand over the life cycle. We then identified all listed apps that once offered social functionality as the participants in the social networking ecosystem. The final dataset includes 1949 apps across different categories at iOS store.

In the second step, we traced the apps' identity (re)projection activities during the 67 months according to their function update history since establishment. This app information, combining with other data dimensions (e.g. app ranking, app rating, user comments), is collected from multiple sources through web crawler, which could be cross-checked against with each other. Specifically, we classified each app update record into one or more categories based on following labels: social function update $(S)$, non-social function update $(N S)$, function maintenance $(M)$ and API connection $(C)$. We did this text categorization task through semi-supervised learning based on BERT model. (Figure 1) In simple words, we firstly randomly select $10 \%$ apps to label their update history manually which constitutes our initial training set to predict the remaining dataset. After the first round of prediction, we second-rounded the manual coding for the top $10 \%$ data with highest uncertainty figured by BERT model, followed by machine prediction. After several rounds of processing, the prediction accuracy reached our expectation and we stopped. We list the typical coding criteria and words for labeling in Table 1.

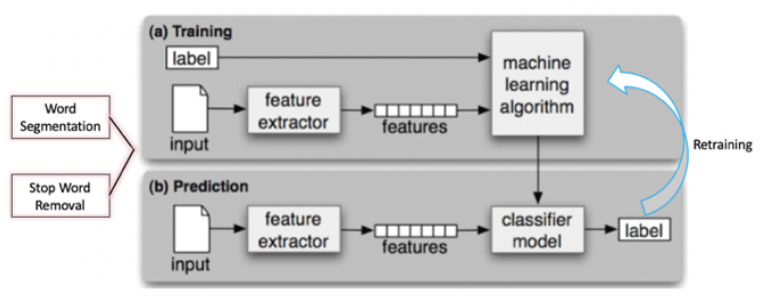

Figure 1. Text classification procedure.

\subsection{Measurement}

We use two proxies to measure the dependent variable app survival according to two proxies. First, we created a dichotomous variable to indicate whether the app is removed (1) or still existed (0) at iOS store at the end of our observation period. We also counted the corresponding total month that an app is available at iOS store as survival duration.

For the main independent variable identity (re)projection, we measure each projection strategy according to the labels we classified before, given the apparent mapping between platform architecture design 
and its function innovation/optimization. Specifically, accumulated boundary resource connection each month is directly measured by the API connection claimed explicitly or implicitly in app update history. Accumulated social function update each month is used as a proxy to indicate architecture assimilating in the social networking ecosystem. Accumulated non-social function update each month is used to measure architecture dissimilating in the social networking ecosystem. Accumulated function maintenance each month is used to indicate architecture maintenance in the ecosystem.

As for the membership of an app claimed by its managers, we use the app category it chooses at iOS store as proxy. As Apple's official website explained, an app should consider its category in terms of three dimensions. First, the category should best describe the main function or subject matter of your app. Second, the category should help identify the user group that will naturally look for an app like yours. Third, the category should contain the same type of apps as yours. Consequently, category indicates target users and a membership of broad groups of platforms with similar core interaction (cf. [31]), with which an app developers attempt to affiliate in the social networking ecosystem. Specifically, there are total 26 categories (e.g. social, health, education, e-commerce) that an app could choose and change during its launch period. We then measure the accumulated number of category change each month as the indicator of membership shift.

In addition, we control multiple variables in the analysis. Network effect refers to the monthly active user and average monthly average use time per user at an app. Portfolio effects refers to the number of sister apps offered by the same developer in each month. Other control variables include app quality in terms of monthly user rating and accumulated user comment number each month, competitive position in terms of monthly ranking in both affiliated category and across categories, average app number in the affiliated category, app age and number of update history before our observation period. The descriptive statistics of those variables are presented in Table 2.

\section{Analysis and Result}

Since our data are censored and nested around different app categories at iOS store, we adopt a Cox proportional hazards $(\mathrm{PH})$ model with a frailty term. The survival we model is the length of time of duration that an app remains in iOS appstore before removing off. The goal is to understand the relationship between the "risk" of experiencing an event ("death" or existing at iOS store) at time $t$ and values of a variety of explanatory variables. A Cox $\mathrm{PH}$ model allows us to handle both categorical and continuous variables without specifying the baseline hazard. By including the frailty term, the model further accounts for unobserved heterogeneity at the app category level. We present the econometric specification as following:

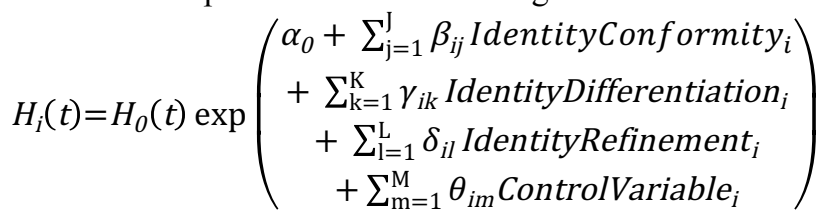

Table 1. Sample text labeling codes.

\begin{tabular}{|l|l|l|}
\hline \multicolumn{1}{|c|}{ Label } & \multicolumn{1}{|c|}{ Overall Criteria } & \multicolumn{1}{c|}{ Sample Codes } \\
\hline Social function update & $\begin{array}{l}\text { Function directly contributes to } \\
\text { user interaction, content sharing, } \\
\text { or content community }\end{array}$ & $\begin{array}{l}\text { Chat; topic; message; friend; emoji; @; sharing; } \\
\text { record; group document; friend circle; video call; } \\
\text { nearby; face-to-face; dating; contacts; reply }\end{array}$ \\
\hline $\begin{array}{l}\text { Non-social function } \\
\text { update }\end{array}$ & $\begin{array}{l}\text { Function does not contribute to } \\
\text { user interaction, content sharing, } \\
\text { or content community directly }\end{array}$ & $\begin{array}{l}\text { Map; trip; health; game; music; dance; wallet; } \\
\text { interface; payment; weather; special effects; filter; } \\
\text { beauty; AR; 3D; geography; travel; shopping }\end{array}$ \\
\hline Function maintenance & Function optimization and bug fix & $\begin{array}{l}\text { Strengthen, optimize; fix; speed; bugs; collapse; } \\
\text { crash; failure; compatibility; fluent; performance }\end{array}$ \\
\hline API connection & $\begin{array}{l}\text { Connecting with other apps or } \\
\text { combing other apps' function }\end{array}$ & $\begin{array}{l}\text { Connect; synchronize; QQ; WeChat; Weibo; } \\
\text { AliPay; third-party sharing; interconnect; Circle } \\
\text { of friends; channeling; skip/jump; inbound links }\end{array}$ \\
\hline
\end{tabular}

Table 2. Sample Descriptive Statistics (N=1917 after dropping left-censoring). 


\begin{tabular}{|c|l|l|l|l|l|}
\hline \multirow{2}{*}{$\begin{array}{c}\text { Identity } \\
\text { conformity }\end{array}$} & API connection & No. of API connection (C) & 2.26 & 1 & 10.10 \\
\cline { 2 - 6 } & $\begin{array}{l}\text { Architecture } \\
\text { assimilation }\end{array}$ & No. of Social function update (S) & 11.84 & 8 & 178.98 \\
\hline $\begin{array}{c}\text { Identity } \\
\text { differentiation }\end{array}$ & $\begin{array}{l}\text { Architecture } \\
\text { dissimilation }\end{array}$ & $\begin{array}{l}\text { No. of Non-social function } \\
\text { update (NS) }\end{array}$ & 14.51 & 9 & 237.87 \\
\hline $\begin{array}{c}\text { Identity } \\
\text { Refinement }\end{array}$ & $\begin{array}{l}\text { Architecture shift } \\
\text { maintenance }\end{array}$ & No. of app category change (CC) & 0.28 & 0 & 0.45 \\
\hline
\end{tabular}

We use the time-invariant explanatory variables by creating accumulated number for main independent variables and the average/median value for other control variables. To test the validity of the result, we also apply other two survival models in this paper, including a discrete-time logic random effects hazard model with time-varying variables and Weibull random effect hazard model. Results are stable across all three models as shown in Table 3. To further check the self-selection issue of app developers in making identity projection decisions, we conduct Heckman two-step test and propensity score matching for the dataset, both of which support the validity of our major finding that identity projection increases the survival likelihood of non-focal platforms in digital ecosystems.

API Connection: Connecting API of focal platforms is associated with significant decrease in the hazard ratio of removing from the iOS store. In particular, change in API connection update is estimated to increase survival time by appropriately $8.04 \%$, supporting $\mathrm{H} 1$.

Architecture Assimilation: Making social functionality update has no significant effect on the survival duration of an app at iOS store. In other words, developing similar technological capabilities (e.g. enhancing social interaction among users) relative to other actors in the social networking ecosystem does not contribute to the app survival. Our estimate allows us to rule out even a pretty small effect of $0.5 \%$ change in survival time. Hence, $\mathrm{H} 2$ is supported. This result is especially interesting and counterintuitive given the fact that more existing and emerging apps across different domains tend to combine social computing technologies as a strategy to build new competitive advantage.

Table 3. app Survival Estimation Result.

\begin{tabular}{|c|c|c|c|}
\hline $\begin{array}{l}\text { Parame } \\
\text { ter }\end{array}$ & $\begin{array}{l}\text { (1) } \\
\text { Cox with } \\
\text { gamma } \\
\text { frailty }\end{array}$ & $\begin{array}{l}\text { (2) } \\
\text { Weibull with } \\
\text { gamma } \\
\text { frailty }\end{array}$ & $\begin{array}{c}(3) \\
\text { Discrete- } \\
\text { time logic }\end{array}$ \\
\hline $\mathrm{C}$ & $0.92^{* *}(.027)$ & $0.92^{* *}(.027)$ & $0.94^{*}(.027)$ \\
\hline $\mathrm{S}$ & $1.01 \quad(.008)$ & $1.01 \quad(.008)$ & $1.00(.008)$ \\
\hline
\end{tabular}

\begin{tabular}{|c|c|c|c|}
\hline NS & $0.98^{* *}(.008)$ & $0.98^{* *}(.008)$ & $0.98^{*}(.009)$ \\
\hline $\mathrm{CC}$ & $1.09 \quad(.070)$ & $1.09 \quad(.070)$ & $1.06 \quad(.070)$ \\
\hline $\mathrm{M}$ & $0.96^{* * *}(.005)$ & $0.96^{* * *}(.005)$ & $0.98^{* *}(.006)$ \\
\hline $\mathrm{U}$ & $0.80^{* * * *}(.034)$ & $0.80^{* * * *}(.034)$ & $0.83^{* * *}(.036)$ \\
\hline $\mathrm{T}$ & $0.83^{* * *}(.031)$ & $0.83^{* * *}(.031)$ & $0.84^{* * * *}(.030)$ \\
\hline ASC & $0.91^{* * * *}(.020)$ & $0.91^{* * * *}(.020)$ & $0.90^{* * * *}(.020)$ \\
\hline $\mathrm{ADC}$ & $1.01 \quad(.003)$ & $1.01 \quad(.003)$ & $1.01 \quad(.003)$ \\
\hline UR & $1.18^{* * * *}(.048)$ & $1.18^{* * * *}(.048)$ & $1.09^{* *}(.044)$ \\
\hline $\mathrm{UC}$ & $0.99 \quad(.000)$ & $0.99 \quad(.000)$ & $1.00 \quad(.000)$ \\
\hline$\overline{C R}$ & $1.30^{* *}(.000)$ & $1.30^{* *}(.000)$ & $1.26^{* * *}(.071)$ \\
\hline OR & $0.87 \quad(.135)$ & $0.87 \quad(.135)$ & (.168) \\
\hline UBO & $0.98 \quad(.012)$ & $0.98 \quad(.012)$ & $0.99 \quad(.013)$ \\
\hline Frailty & $0.11^{* * * *}(.055)$ & $0.11^{* * *}(.055)$ & $0.10^{* * *}(.051)$ \\
\hline$\overline{L L}$ & -4039 & -1253 & -3062 \\
\hline
\end{tabular}

Architecture Dissimilation: As expected, by differentiating the technological capabilities from other similar apps in the social networking ecosystem through innovating non-social functionality (e.g. e-commerce, map, music, game) is strongly associated with the survival duration of an app at iOS store. Specifically, apps with one more update of non-social function tend to survive $2.4 \%$ longer at iOS store $(P=0.005)$. These findings support $\mathrm{H} 3$.

Membership Shift: We also find no evidence that frequent change of app membership across different categories (e.g. social, music, book, travel, health) defined at iOS store will be association with an app's survival duration, indicating the potential cancelling effect between the advantages and drawbacks brought by membership shift. Hence, $\mathrm{H} 4$ is supported.

Architecture Maintenance: As expected, we also find that reinforcing existing projected identity through functionality optimization is associated with a strong positive effect on the survival time of an app at iOS store. In particular, apps with one more update of function maintenance are estimated to survival approximately $4.6 \%$ more months at iOS store. Hence, H5 is clearly supported.

Control Variables: Network effect is positively associated with the survival time of an app, both from 
user base and user engagement. Number of sister apps produced in same category positively contribute to the survival duration, indicating the potential knowledge and resource sharing among similar apps produced by the same developer. In addition, more controversial apps with relatively lower rating seems survival longer in the matured digital market. With regard to the competitive position, competitive apps are positively associated with survival duration. In particular, only the ranking in the category that the app affiliates is significant comparing with its overall ranking across categories, showing the importance of withinmembership competition for app survival in matured ecosystems.

\section{Discussion and Conclusion}

Our paper contributes to the platform literature, especially platform identity, by conceptualizing three identity projection strategies, namely identity conformity, identity differentiation and identity refinement. Based on the further testing of their relationship with non-focal platform survival in digital ecosystems, we discuss each of them as following.

First, identity conformity refers to the commitment to the identity-related expectations from ecosystem members about "appropriate" and "inappropriate" activities and positions in the ecosystem. Prior study emphasized the conformity towards the membership of broad groups of like organizations, mainly because such category-based expectation constructs the taken-forgranted practices and logics which are the key sources of legitimacy [17, 9]. However, our results reveal that non-focal actors are more sensitive to focal platforms' expectations in digital ecosystem context, manifested in the significant effect of API connection on app survival. At the core of this difference, focal actors set and enforce the governance rules, alignment structure and reap the lion's share of gains in a platform ecosystem [26]. In this regard, the endorsement from focal actors become more vital for firm survival than other similar non-focal actors in the ecosystem. Moreover, the insignificant association between architecture assimilation and app survival indicates the unbounded innovation space $[30,38]$ in platform ecosystem context, where actors are more inclusive for uncertainty and ambiguity due to the fluid and evolving scope, feature, and value of digital offerings [24]. As such, "the liability of newness" [45] highlighted in industrial context is no longer an issue for platform businesses to gain legitimacy, which do not need to bound themselves within a particular membership [46].

Second, identity differentiation refers to differentiating the platform identity from other similar actors within an ecosystem. Extant research has suggested that external stakeholders accept some degree of variation in conformity to their expectations [9] as long as the organization is believed to be "focused enough" ([47], p. 69). In platform ecosystem context, this degree of freedom to project and enact identity discretionarily becomes higher and weighted more importantly for firm development, manifested in the significant relationship between architecture dissimilation and app survival. Since digital ecosystems are open-ended with low cost to participate [24], an ecosystem actor will confront less predefined competitors with diverse goals, motives and capabilities. In this condition, focal actors benefit from attracting heterogeneous innovation agency [38], leading to escalating rivalry among non-focal actors. As such, platform ecosystem members value more on novelty and distinctiveness than conformity. At the same time, our study also indicates the necessity to build distinctiveness upon the focused core interaction (cf. [31]) in platform context. Since the core interaction defines the fundamental value to participants and attracts most users to the platform in the first place, arbitrary shift of platform membership characterized by similar core interaction propositions will counteract the potential novelty it may bring, leading to insignificant effect on app survival in our results. In the end, we support to mirror the layered platform architecture design when projecting platform identity - that is, developing architecture dissimilation on top of core membership.

Third, identity refinement refers to reinforcing existing projected identity without major change. While organization identity literature tends to discuss the "enduring" or "continuity" ends underlying such refinement strategy which help to yield enhanced legitimacy and higher survival chances $[36,20]$, it starts with a totally different logic in platform ecosystem context, namely "winner-take-all" [48] and "get-bigfast" [21]. As such, platform actors are forced to refine their identity in case of losing position when confronting fierce competition from emerging actors. In this regard, refinement strategy rather implies a volatile nature of identity in platform ecosystems, which has to be elaborately defended in order to maintain the established legitimacy. Accordingly, successful refinement of platform identity will highly increase the chances of survival which is shown in the effect of architecture maintenance on app survival.

Combining the three identity projection strategies together, platform identity seems to be characterized by dynamics which involves periods of ongoing change punctuated by episodes of perceived stability. In Huang et al. 's [49] work, it is marked as a continuous shift process through "recurring definition of what they do and who they are" (p. 9). Ultimately, non-focal actors in 
platform ecosystems may "defend" or "betray" their existing identity in order to cater to competing market imperatives-that is, the expectation of what an organization should be in order to be competitive with other actors [22, 18]. To fully unpack this dynamic nature of platform identity, we ask further investigation to reinvent organizational identity research in a digital world.

In addition, we contribute to the understanding of platform competition in an increasingly prevalent scenario - mature digital markets. With the stabilization of various digital markets dominated by early platform giants (e.g. Google, apple, Amazon), firms are more frequently forced to engage in multiple established ecosystems in order to seek for new growth opportunities. As such, even those focal actors in the native ecosystems may descend to be non-focal during the cross-ecosystem participation. Identity recognition in an ecosystem thus become more vital than ever before. Our study therefore echoes to the recent call for providing practical implications to those emerging and incumbent firms who attempt to participate across different digital ecosystems in the digital age [27].

Despite valuable contributions, our study has some limitations which provide opportunities for future studies. First, we focus only on the social networking ecosystem at iOS store. Since this is a pure digital space, we may expect different identity projection patterns and impact on platform development in other contexts (e.g. platforms crossing digital and physical space like Uber and Airbnb). We therefore call for future studies to replicate our findings in different contexts to confirm generalizability. Second, we locate the study in matured digital markets. However, it does not exhaust the need to understand platform identity in emerging and rapidgrowing contexts, especially its role in facilitating digital transformation process in numerous industrial markets. We therefore hope that our research can stimulate further studies in the area and serves as a prelude to the understanding of platform identity in the digital age when participation beyond single ecosystem becomes compulsory for survival and further growth of every digital business.

\section{References}

[1] Raz O, Gloor PA., "Size Really Matters-New Insights for Start-Ups' Survival”, Management Science, 53(2), 2007, pp.169-177.

[2] Kazan, Erol, Chee-Wee Tan, Eric TK Lim, Carsten Sørensen, Jan Damsgaard., "Disentangling Digital Platform Competition: The Case of UK Mobile Payment Platforms", Journal of Management Information Systems, 35(1), 2018, pp. 180-219.

[3] Li, Shanling, Jennifer Shang, Sandra A. Slaughter., "Why Do Software Firms Fail? Capabilities, Competitive
Actions, and Firm Survival in the Software Industry from 1995 To 2007", Information Systems Research, 21(3), 2010, pp. 631-654.

[4] Lotti F, Santarelli E, Vivarelli M., "Does Gibrat's Law Hold Among Young, Small Firms?", Journal of Evolutionary Economics, 13(3), 2003, pp. 213-35.

[5] Jacobides, Michael G., Carmelo Cennamo, Annabelle Gawer., "Towards a Theory of Ecosystems", Strategic Management Journal, 39(8), 2018, pp. 2255-2276.

[6] Selander, Lisen, Ola Henfridsson, Fredrik Svahn., "Capability Search and Redeem across Digital Ecosystems", Journal of Information Technology, 28(3), 2013, pp. 183-197.

[7] Cennamo, Carmelo, Hakan Ozalp, Tobias Kretschmer., "Platform Architecture and Quality Trade-Offs of Multihoming Complements", Information Systems Research, 29(2), 2018, pp. 461-478.

[8] Ghazawneh, A., Henfridsson, O., "Balancing Platform Control and External Contribution in Third-Party Development: The Boundary Resources Model", Information Systems Journal,23(2), 2013, pp.173-192.

[9] Navis, Chad, Mary Ann Glynn., "Legitimate Distinctiveness and the Entrepreneurial Identity: Influence on Investor Judgments of New Venture Plausibility", Academy of Management Review, 36(3), 2011, pp. 479-499.

[10] Delmar, Frédéric, Scott Shane., "Legitimating First: Organizing Activities and the Survival of New Ventures", Journal of Business Venturing, 19(3), 2004, pp. 385-410.

[11] Zimmerman, Monica A., Gerald J. Zeitz., "Beyond Survival: Achieving New Venture Growth by Building Legitimacy", Academy of Management Review, 27(3), 2002, pp. 414-431.

[12] Tiwana, Amrit., "Evolutionary Competition in Platform Ecosystems", Information Systems Research, 26(2), 2015, pp. 266-281.

[13] Geroski, Paul A., "What Do We Know about Entry?", International Journal of Industrial Organization, 13(4), 1995, pp. 421-440.

[14] Gioia, Dennis A., Majken Schultz, Kevin G. Corley., "Organizational Identity, Image, and Adaptive Instability", Academy of Management Review, 25(1), 2000, pp. 63-81.

[15] Tripsas, Mary., "Technology, Identity, and Inertia through the Lens of "The Digital Photography Company"", Organization Science, 20(2), 2009, pp. 441460.

[16] Dutton, Jane E., Janet M. Dukerich., "Keeping an Eye on the Mirror: Image and Identity in Organizational Adaptation", Academy of Management Journal, 34(3), 1991, pp. 517-554.

[17] Anthony, Callen, Mary Tripsas., "Organizational Identity and Innovation", The Oxford Handbook of Organizational Identity, 2016, pp. 417-435.

[18] Gioia, Dennis A., Shubha D. Patvardhan, Aimee L. Hamilton, Kevin G. Corley., "Organizational Identity Formation and Change", Academy of Management Annals, 7(1), 2013, pp. 123-193. 
[19] Gawer, Annabelle, Rebecca Henderson., "Platform Owner Entry and Innovation in Complementary Markets: Evidence from Intel", Journal of Economics \& Management Strategy, 16(1), 2007, pp. 1-34.

[20] Whetten, David A., "Albert and Whetten Revisited: Strengthening the Concept of Organizational Identity", Journal of Management Inquiry, 15(3), 2006, pp. 219-234.

[21] Cennamo, C., "Competing in Digital Markets: A Platform-Based Perspective", Academy of Management Perspectives, 35(2), 2021, pp. 265-291.

[22] de Reuver, M., Sørensen, C., Basole, R. C., "The Digital Platform: A Research Agenda", Journal of Information Technology, 33(2), 2017, pp. 124-135.

[23] von Briel, Frederik, Jan Recker, Lisen Selander, Sirkka L. Jarvenpaa, Philipp Hukal, Youngjin Yoo, Julian Lehmann et al., "Researching Digital Entrepreneurship: Current Issues and Suggestions for Future Directions", Communications of the Association for Information Systems, 48(1), 2021, pp. 33.

[24] Yoo, Youngjin, Ola Henfridsson, Kalle Lyytinen., "Research Commentary_-The New Organizing Logic of Digital Innovation: An Agenda for Information Systems Research", Information Systems Research, 21(4), 2010, pp. 724-735.

[25] Arthur, W. Brian., The Nature of Technology: What It Is and How It Evolves, Simon and Schuster, 2009.

[26] Adner, Ron. "Ecosystem as Structure: An Actionable Construct for Strategy", Journal of Management, 43(1), 2017, pp. 39-58.

[27] Hein, A., Schreieck, M., Riasanow, T., Setzke, D. S., Wiesche, M., Böhm, M., Krcmar, H., "Digital Platform Ecosystems", Electronic Markets, 30(1), 2020, pp. 8798.

[28] Gawer, Annabelle., Michael A. Cusumano. Platform Leadership: How Intel, Microsoft, and Cisco Drive Industry Innovation. Vol. 5. Boston, MA: Harvard Business School Press, 2002.

[29] Leong, Carmen, Shan L. Pan, Dorothy E. Leidner, JinSong Huang., "Platform Leadership: Managing Boundaries for the Network Growth of Digital Platforms", Journal of the Association for Information Systems, 20(10), 2019, pp. 1531-1565.

[30] Henfridsson, Ola, Joe Nandhakumar, Harry Scarbrough, Nikiforos Panourgias., "Recombination in the OpenEnded Value Landscape of Digital Innovation", Information and Organization, 28(2), 2018, pp. 89-100.

[31] Parker, Geoffrey G, Marshall W. Van Alstyne, Sangeet Paul Choudary., Platform Revolution: How Networked Markets Are Transforming the Economy and How to Make Them Work for You, WW Norton \& Company, 2016.

[32] Boudreau, Kevin, Karim Lakhani., "How to Manage Outside Innovation", MIT Sloan Management Review, 50(4), 2009, pp. 69.

[33] Suchman, Mark C., "Managing legitimacy: Strategic and Institutional Approaches", Academy of Management Review, 20(3), 1995, pp. 571-610.
[34] Deephouse, David L., "Does Isomorphism Legitimate?", Academy of Management Journal, 39(4), 1996, pp. 1024-1039.

[35] Brewer, Marilynn B., "The Social Self: On Being the Same and Different at the Same Time", Personality and Social Psychology Bulletin, 17(5), 1991, pp. 475-482.

[36] Drori, Israel, Benson Honig, Zachary Sheaffer., "The Life Cycle of an Internet Firm: Scripts, Legitimacy, and Identity", Entrepreneurship Theory and Practice, 33(3), 2009, pp. 715-738.

[37] Zook, Matthew., The Geography of the Internet Industry: Venture Capital, Dot-Coms, and Local Knowledge, John Wiley \& Sons, 2008.

[38] Nambisan, Satish, Kalle Lyytinen, Ann Majchrzak, Michael Song., "Digital Innovation Management: Reinventing Innovation Management Research in A Digital World", MIS Quarterly, 41(1), 2017, pp. 223238.

[39] Cloutier, Charlotte, Davide Ravasi., "Identity Trajectories: Explaining Long-Term Patterns of Continuity and Change in Organizational Identities", Academy of Management Journal, 63(4), 2020, pp. 1196-1235.

[40] Livengood, R. Scott, Rhonda K. Reger., "That's Our Turf! Identity Domains and Competitive Dynamics", Academy of Management Review, 35(1), 2010, pp. 48-66.

[41] Hsu, Greta, Michael T. Hannan., "Identities, Genres, and Organizational Forms", Organization Science, 16(5), 2005, pp. 474-490.

[42] Oestreicher-Singer, Gal, Lior Zalmanson., "Content or Community? A Digital Business Strategy for Content Providers in the Social Age", MIS Quarterly, 37(2), 2013, pp. 591-616.

[43] Zhu, F., K. G. Palepu, B. Y. Cao, D. H. Lau., "Pinduoduo", Harvard Business School Case, 2019, pp. 620-040.

[44] Liu, Shiyuan, Nandhakumar, Joe, Henfridsson, Ola., "Balancing User Base and User Stickiness in Platform Scaling", In Proceedings of the 27th European Conference on Information Systems (Stockholm, Sweden), 2019, Research-in-Progress Papers.

[45] Stinchcombe, A. L., "Social Structure and Organizations", In J. C. March (Ed.), Handbook of Organizations, Chicago: Rand McNally, 1965, pp. 142193.

[46] Thomas, L. D, Ritala, P., "Ecosystem Legitimacy Emergence: A Collective Action View", Journal of Management, 2021, DOI: 0149206320986617.

[47] Vergne, J-P, Tyler Wry., "Categorizing Categorization Research: Review, Integration, and Future Directions", Journal of Management Studies, 51(1), 2014, pp. 56-94.

[48] Eisenmann, Thomas, Geoffrey Parker, Marshall W. Van Alstyne., "Strategies for Two-Sided Markets", Harvard Business Review, 84(10), 2006, pp. 92.

[49] Huang, Jimmy, Ola Henfridsson, Martin J. Liu, Sue Newell., "Growing On Steroids: Rapidly Scaling the User Base of Digital Ventures through Digital Innovaton", MIS Quarterly, 41(1), 2017. 